\title{
Comparison of ALE finite element method and adaptive smoothed finite element method for the numerical simulation of friction stir welding
}

\author{
A.A. van der Stelt*, T.C. Bor ${ }^{\dagger}$, H.J.M. Geijselaers ${ }^{\dagger}$, \\ W. Quak ${ }^{\dagger}$, R. Akkerman ${ }^{\dagger}$ and J. Huétink ${ }^{\dagger}$ \\ * Materials innovation institute (M2i), P.O. Box 5008, 2600 GA Delft, the Netherlands \\ ${ }^{\dagger}$ Faculty of Engineering Technology University of Twente, PO Box 217, 7500 AE Enschede, The Netherlands
}

\begin{abstract}
In this paper, the material flow around the pin during friction stir welding (FSW) is simulated using a 2D plane strain model. A pin rotates without translation in a disc with elasto-viscoplastic material properties and the outer boundary of the disc is clamped. Two numerical methods are used to solve this problem and an analytical solution is derived. The analytical model is complementary to validate the two numerical methods, i.e. the arbitrary Lagrangian-Eulerian (ALE) method and the adaptive smoothed finite elements method (ASFEM).
\end{abstract}

Keywords: Friction stir welding, analytical solution, arbitrary Lagrangian-Eulerian method, adaptive smoothed finite elements method, elasto-viscoplastic

PACS: $02.70 . \mathrm{Dh}, 46.15 .-\mathrm{x}, 46.25 .-\mathrm{y}, 46.35 .+\mathrm{z}$

\section{INTRODUCTION}

Friction stir welding (FSW) is a relatively new solid-state joining technology for metals, see Figure 1 (1) for a schematic representation of the process. It shows no solidification-related joint imperfections which makes it suitable for hard-toweld highly alloyed aerospace aluminum grades, like AA 2xxx and 7xxx. The weld properties strongly depend on the welding conditions. Important process parameters are the welding speed, the tool rotation rate and the applied down force. Also the type of tool and material grade play an important role. A thorough understanding of the material flow around the pin of the rotating tool moving through the work piece is required to improve the weld quality and speed up the welding process. Various approaches exist to simulate the material flow around the tool pin [1], [2]. However, it is not reported that these models are validated with an analytical model which describes the elasto-viscoplastic material flow around the pin.

Many advanced numerical models for simulating and optimizing the material flow in FSW use the arbitrary Lagrangian-Eulerian (ALE) method. This method is known for its capability of describing large deformations and the deformation of free boundaries. This specific property makes it suitable for modeling the material flow of friction stir welding while the tool is moving through the work piece. Unfortunately, inaccuracies are generated during the convection of history dependent material parameters. An example is the local yield stress, which is not only dependent on the local temperature, but also on the degree of prior plastic deformation and/or (dynamic) recrystallization.

An alternative numerical method is the meshfree method, which is relatively new and has been used seldom in the modeling of the FSW process up to now. A typical example in the simulation of material flow during 2D FSW is given by Alfaro et al. [3]. They used this method because it alleviates the difficulties due to the treatment of convective terms assuming viscoplastic material behavior. A method which has similar properties as the meshfree method is the adaptive smoothed finite elements method (ASFEM) developed by Quak et al. [4]. Both methods use nodal integration and they need only nodes as an input for the generation of a numerical model, so they do not require an initial mesh.

In this paper, an ALE method and the ASFEM method are compared with an analytical solution for a problem which describes the elasto-viscoplastic material behavior around a rotating pin. In this way the accuracy of both methods is determined. Interesting parameters are the shear stress distribution, the viscoplastic bandwidth around the pin and the magnitude of the elastic deformation around the viscoplastic domain. 


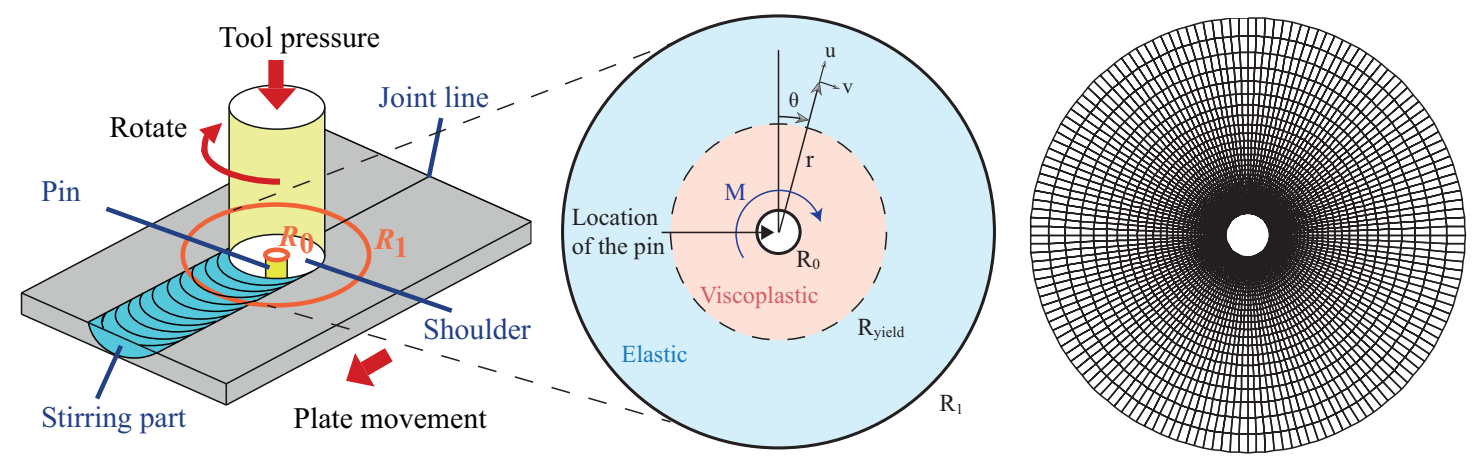

FIGURE 1. (1) Schematic representation of friction stir welding. (m) 2D plane strain problem of FSW without plate movement. (r) Nodes and elements of the discretized problem for a coarse mesh with 6080 nodes.

\section{MODEL DEVELOPMENT}

The 2D plane strain problem studied in this work is shown in Figure $1(\mathrm{~m})$. It represents the area around a pin with radius $R_{0}$ during FSW when there is no translational velocity of the plate. The plate is represented by a circular region with radius $R_{1}$ and it estimates the viscoplastic domain size around the pin. This stationary state where the plate is not moving is equivalent to friction stir spot welding. A moment $M$ is applied at the inner radius $R_{0}$ of the disc for representing the rotation of the pin. The disc is clamped at the outer radius $R_{1}$. This radius should be large enough such that the transition from viscoplastic to elastic deformation can be clearly identified. The solution of the problem depends only on the radius $r$ and not on the angle $\theta$ because the problem is axisymmetric. Displacements in the domain are only in circumferential direction $v$ and not in the radial direction $u$.

The problem is solved for an isothermal process which includes the Sellars-Tegart hardening law for Von Mises elasto-viscoplasticity neglecting the inertia terms. The hardening law depends on the plastic strain rate and not on the plastic strain itself. This is a valid assumption for high temperature processes with large deformations. It is often used for the simulation of aluminium extrusion processes at high temperatures.

First, an analytical solution is found. Next, the numerical methods solve the weak form of the discretized force and momentum balance equations. The discretized domain for the coarse mesh is shown in Figure 1 (r) and it has 6080 nodes. Finally, the results of the three different methods are compared with each other. The three different solution methods are explained in the following sections, i.e the analytical, the ALE and the ASFEM method, respectively.

\section{Analytical method}

An analytical solution of the problem is derived for the elasto-viscoplastic material model. This derivation is performed in three steps. First, a derivation is performed for the elastic material model and secondly for the viscoplastic material model. Together they form a solution for the elasto-viscoplastic material model.

The applied moment $M$ on the inner radius of the geometry determines the stress $\tau$ for all three cases. If force and moment equilibria are considered, the shear stress depends on radius $r$ as:

$$
\tau=\frac{M}{2 \pi r^{2}} .
$$

First, it is assumed that the material responds fully elastically. Assuming a rotational symmetric displacement field, the relation between $\gamma_{r \theta}$ and the displacement field can be determined:

$$
\gamma_{r \theta}=\frac{1}{r} \frac{\partial u}{\partial \theta}+\frac{\partial v}{\partial r}-\frac{v}{r} .
$$

It follows easily from $\tau=G \gamma$ that the rotational deformation $\phi$ is: 


$$
\phi=\frac{v}{r}=\frac{M}{4 \pi G}\left(\frac{1}{r^{2}}-\frac{1}{R_{1}^{2}}\right),
$$

assuming a moment $M$ at $R=R_{0}$ at the boundary with the pin and no displacement at $R=R_{1}$ (clamped).

Secondly, it is assumed that the material is fully viscoplastic. In a similar fashion, using the time derivative of Equation (2) and the Sellars-Tegart law, the strain rate distribution can be determined. The Sellars-Tegart law reads:

$$
\tau_{\text {viscoplastic }}(\dot{\kappa})=s_{m} \operatorname{arcsinh}\left(\left(\frac{\dot{\kappa}+\dot{\kappa_{0}}}{A} e^{Q / R T}\right)^{\frac{1}{m}}\right), \text { and } \dot{\kappa}=\sqrt{\frac{1}{3}} \dot{\gamma},
$$

where $s_{m}, m$ and $A$ are material dependent strain sensitivity parameters, $Q$ is the apparent activation energy of the process during viscoplastic flow, $R$ is the universal gas constant, $T$ is the absolute temperature and $\dot{\kappa}_{0}$ is the initial equivalent viscoplastic strain rate. Then the rotational deformation speed $\omega$ follows:

$$
\omega=\frac{\dot{v}}{r}=\sqrt{3} A e^{-Q / R T} \int_{r}^{R_{1}} \frac{\left(\sinh \left[\sqrt{3} \frac{M}{2 \pi r^{2}} \frac{1}{S_{m}}\right]\right)^{m}}{r} d r-\sqrt{3} \dot{\kappa}_{0}\left(\ln \left(R_{1}\right)-\ln (r)\right),
$$

assuming a moment $M$ at $R=R_{0}$ at the boundary with the pin, and no rotation velocity at $R=R_{1}$ (static transition).

In real cases, a viscoplastic region will develop close to the pin, where high shear stresses are present. Further away, the material will respond elastically. The transition radius, $R_{\text {yield }}$, is located there where the Von Mises stress $\tau_{V M}$ equals the yield stress $\tau_{\text {yield }}$ for $\dot{\kappa}=0$. It holds that

$$
\begin{gathered}
\tau_{V M}=\tau \sqrt{3}= \begin{cases}\tau_{\text {elastic }} \sqrt{3} & \text { if } \tau_{V M} \leq \tau_{\text {yield }} \\
\tau_{\text {viscoplastic }} \sqrt{3} & \text { if } \tau_{V M}>\tau_{\text {yield }}\end{cases} \\
R_{\text {yield }}=\sqrt{\frac{\sqrt{3} M}{2 \pi \tau_{\text {yield }}}}
\end{gathered}
$$

The viscoplastic solution is used for the inner part of the disc between $R_{0}$ and $R_{\text {yield }}$ and the elastic solution is used for the outer part of the disc between $R_{\text {yield }}$ and $R_{1}$.

\section{Numerical methods}

\section{Arbitrary Lagrangian-Eulerian method}

The ALE method used in this work is programmed in the in-house DiekA simulation software. It employs the so called semi-coupled formulation with a predictor and a corrector step [5], [6]. In this formulation an updated Lagrangian step is followed by a second step projecting the mesh back on the old domain, excluding the free surface.

\section{Adaptive smoothed finite elements method}

The ASFEM method is based on the approach developed by Quak et al. [4]. In their software a numerical strategy is proposed which is called the method of adaptive smoothed finite elements. This method possesses some interesting properties for the simulation of metal forming processes, such as locking-free behavior, distortion insensitivity and good computational efficiency. Moreover, due to the definition of the smoothed field, it is possible to revise the mesh without mapping the history dependent material parameters. An updated-Lagrangian implementation is used, and an algorithm to maintain details on the boundary. 
TABLE 1. Material parameters for AA6063[7]

\begin{tabular}{lrr}
\hline & Symbol & Value \\
\hline Elastic properties & $E[\mathrm{MPa}]$ & 40000 \\
$(T=823 \mathrm{~K})$ & $v[-]$ & 0.4995 \\
\hline Plastic properties & $s_{m}[\mathrm{MPa}]$ & 25 \\
& $m[-]$ & 5.385 \\
& $A[1 / \mathrm{s}]$ & $5.91 \cdot 10^{9}$ \\
& $Q[\mathrm{~J} / \mathrm{mol}]$ & $1.4155 \cdot 10^{5}$ \\
\hline
\end{tabular}

\section{RESULTS AND DISCUSSIONS}

The rotation speed caused by a rotating pin with radius $R_{0}=2.5 \mathrm{~mm}$ in a circular elasto-viscoplatic material with radius $R_{1}=25 \mathrm{~mm}$ is calculated analytically and shown in Figure 2 (1). This is for an applied moment of $M=844.4$ $\mathrm{Nmm}$ which results in a rotating speed of the pin of $\omega=500 \mathrm{rpm}$ using the material parameters shown in Table 1. Following the procedure outlined above, the elastic rotation field $\phi$ and the $R_{\text {yield }}$ can also be determined from Equations (1) to (7); see Figure 2 (r).

Subsequently, these fields are determined with both numerical methods. As boundary condition, the rotation speed of the pin at $R=R_{0}$ is used instead of the applied moment $M$ for computational reasons. The rotation speed boundary condition is calculated by the analytical method. Four simulations are performed, i.e. 2 for the ASFEM method and 2 for the ALE method. The first simulation has a coarse node distribution of 6080 nodes and the second simulation has refinements near the pin and has 12160 nodes. The purpose is to compare the stress and viscoplastic strain rate distribution of the three different methods. This is only done for the finest node distribution. Finally, the shear stress distribution is plotted and the $R_{\text {yield }}$ is compared for all four simulations.

\section{Deformation and deformation rate}

The applied moment $M=844.4 \mathrm{Nmm}$ and a yield stress $\tau_{\text {yield }}=6.60 \mathrm{~N} / \mathrm{mm}^{2}$ for $\dot{\kappa}=01 / \mathrm{s}$ result in a yield radius of $R_{\text {yield }}=6.08 \mathrm{~mm}$. This separation between viscoplastic and elastic solution is shown in Figure 2 . From $R_{0}=2.5$ $\mathrm{mm}$ to $R_{\text {yield }}=6.08 \mathrm{~mm}$, there is a rotation speed for the viscoplastic behavior and between $R_{\text {yield }}=6.08 \mathrm{~mm}$ and $R_{1}=25 \mathrm{~mm}$ there is a static elastic rotation. A maximum rotation speed is reached of $\omega=500 \mathrm{rpm}$ at $R=R_{0}$ and the maximum rotation is $\phi=7.7 \cdot 10^{-3}$ degrees in the elastic domain at $R=R_{\text {yield }}$. High rotational speeds are observed close to the rotating pin up to approximately $R \approx 3.2 \mathrm{~mm}$ in this case. Furthermore, the elastic rotations are very small.

\section{Stress and viscoplastic shear rate distribution}

The shear stress distribution for the three methods are shown in Figure 3 (1). Both numerical methods use 12,160 nodes for these results; the ALE method has 12,008 elements. Both numerical methods follow the curve of the analytical solution closely. However, the ASFEM method has some difficulties in obtaining the high shear stresses near the pin at $R=R_{0}$. In Figure $3(\mathrm{r})$, there are larger differences between the analytical solution and the ASFEM solution. This can be explained from the functional relation between $\tau$ and $\dot{\kappa}$ from Equation (4). In general, the magnitude of $\dot{\kappa}$ changes rapidly for a small change in magnitude of $\tau$ near the pin at $R=R_{0}$. The global trend as seen from Figure $3(\mathrm{r})$ is that the ALE method is more accurate than the ASFEM method for the viscoplastic strain rate.

The shear stress distribution for the fine node distribution using the ASFEM method is shown in Figure 4. It is the refined region near the pin. Maximum and minimum shear stresses are of equal absolute magnitude. This corresponds with the axisymmetric setup of the problem.

Finally, the yield radii of all four simulations are plotted in Figure 4. The second bar represent the radius of the last viscoplastic node, the first bar represents the radius of the previous node and the third bar for the next node. A 

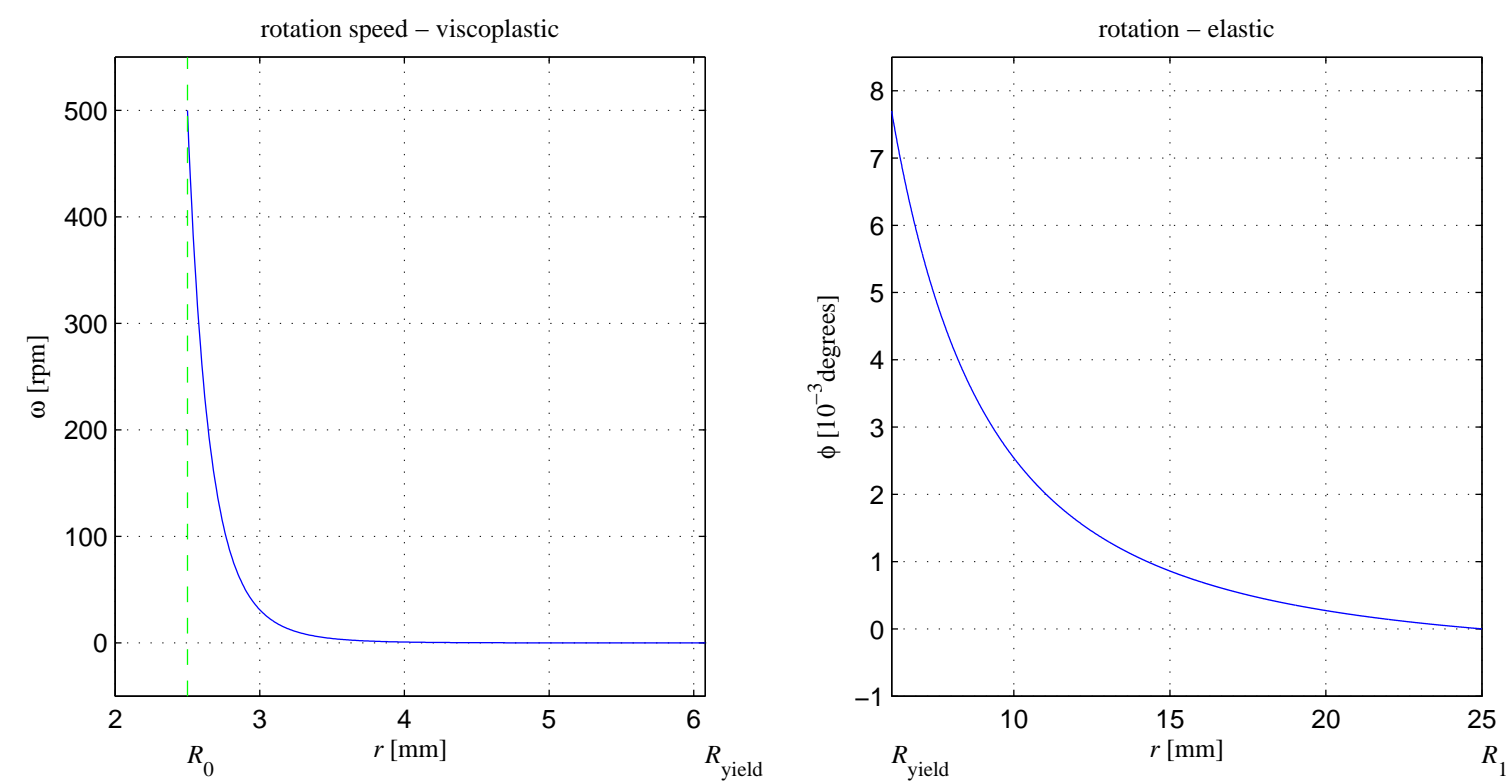

FIGURE 2. (1) Analytical solution of the stationary rotation speed. (r) Analytical solution of the elastic rotation.
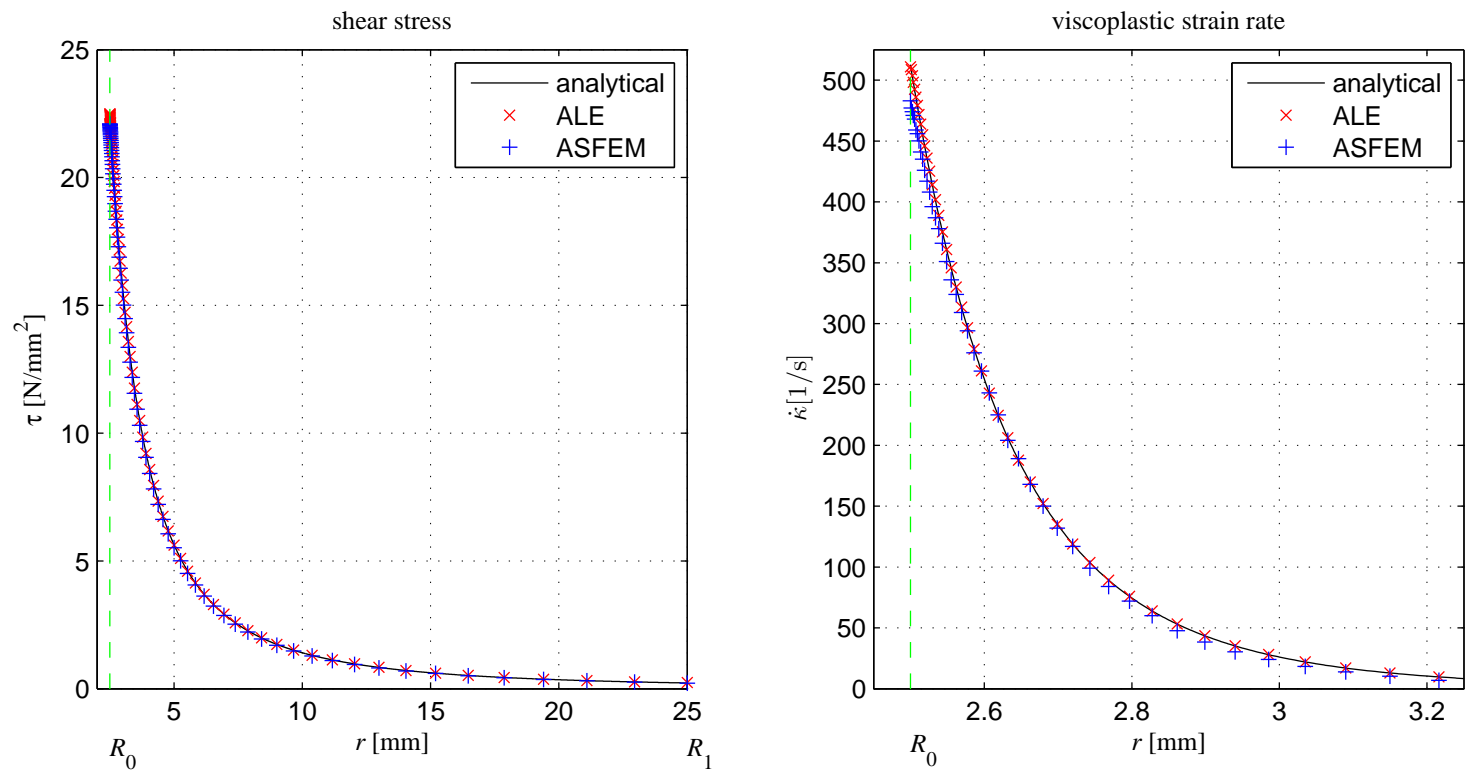

FIGURE 3. (1) Analytical and numerical solution of the shear stress distribution. (r) Analytical and numerical solution of the viscoplastic strain rate distribution.

good prediction is performed for the last three simulations. The $R_{\text {yield }}$ is very close to $R_{\text {yield }}^{\text {analytical }}$. However, for the ASFEM simulation with a coarse node distribution the solution is not correct. The next node is closer to $R_{\text {yield }}^{\text {analytical }}$ and should have been selected as $R_{\text {yield }}$. 

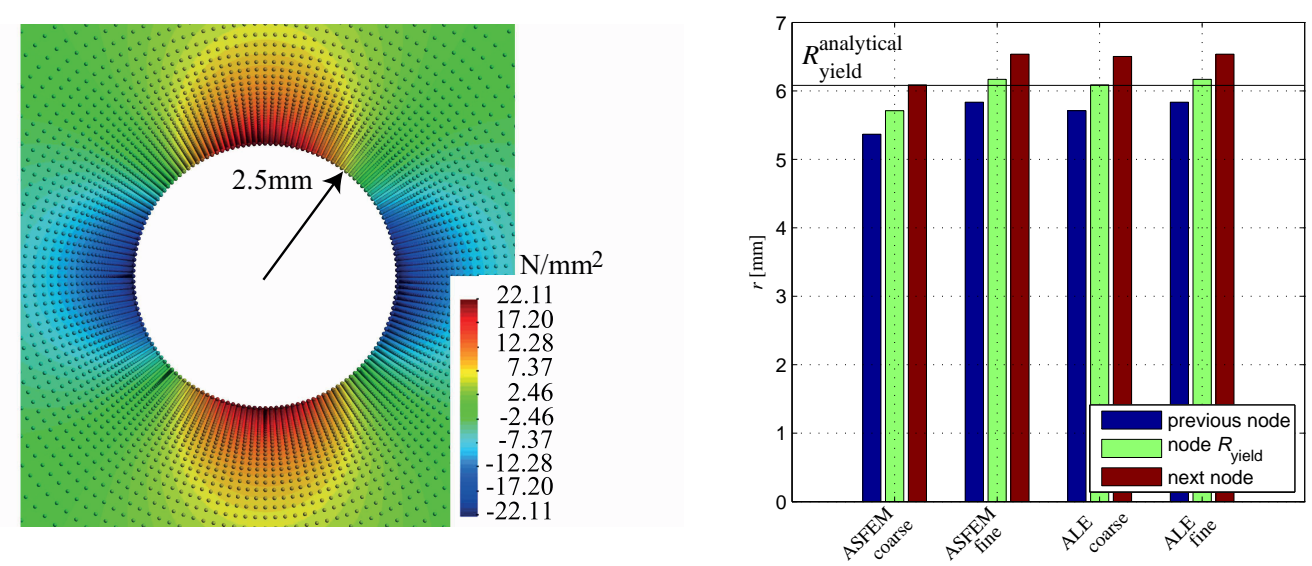

FIGURE 4. (1) Shear stress distribution near the pin using the ASFEM method. (r) Yield radius according different node distributions and numerical methods.

\section{CONCLUSIONS}

In this paper, a validation is performed for two numerical methods applied on a friction stir welding problem. The ALE and ASFEM methods are compared with an analytical model. Both methods are capable of capturing the shape of the stress and viscoplastic strain rate distribution. The stress distribution is accurately predicted by both methods. However, the ALE method is preferred above the ASFEM method for predicting the viscoplastic strain rate, since it is more accurate. Both methods give a good impression of the size of the viscoplastic bandwidth.

\section{ACKNOWLEDGMENTS}

This research was carried out under project number MC8.07290 in the framework of the Research Program of the Materials innovation institute M2i (www.m2i.nl), the former Netherlands Institute for Metals Research.

\section{REFERENCES}

1. R. Mishra, and M. Mahoney, Friction stir welding and processing, ASM International, 2007.

2. D. Lohwasser, and Z. Chen, Friction stir welding: From basics to applications, Woodhead Publishing Ltd, 2009.

3. I. Alfaro, G. Racineux, A. Poitou, E. Cueto, and F. Chinesta, International Journal of Material Forming 2, 225-234 (2009).

4. W. Quak, and A. van den Boogaard, Proceedings of ESAFORM (2011).

5. P. van der Helm, J. Huétink, and R. Akkerman, International Journal for Numerical Methods in Engineering 41, 1057-1076 (1998).

6. H. Wisselink, and J. Huétink, Journal of Materials Processing Technology 148, 328-341 (2004).

7. J. Lof, Developments in finite element simulations of aluminium extrusion., Ph.D. thesis, University of Twente, Enschede (2000). 